INVITED PAPER

\title{
Investigating the maladaptive cortical reorganization in the human auditory cortex using magnetoencephalography
}

\author{
Hidehiko Okamoto* \\ Department of Physiology, School of Medicine, International University \\ of Health and Welfare, \\ 4-3 Kozunomori, Narita, 286-8686 Japan
}

\begin{abstract}
Non-invasive neuroimaging techniques have revealed that not only child brains but also adult brains can be reorganized in human. The cortical reorganization is usually beneficial such as an increased auditory cortical representation in professional musicians. On the other hand, maladaptive cortical reorganization in the auditory cortex can lead to hearing disorders such as tinnitus and hyperacusis. We tried to non-invasively visualize the pathological neural activity in the human auditory cortex and to reverse maladaptive cortical reorganization by suitable behavioral training to decrease detrimental auditory symptoms. Here, we report our previous studies that measured the neural activity in the auditory cortex of hearing impaired people using magnetoencephalography. The results obtained indicated that hearing impairments were related to the reorganization of the auditory neural pathway and that sound therapy was an effective approach for sudden sensorineural hearing loss. Visualization of the healthy and pathological brain activity by non-invasive neuroimaging techniques can lead to the development of a new clinical approach for those affected.
\end{abstract}

Keywords: Brain, Cortical plasticity, Human auditory cortex, Rehabilitation

PACS number: 43.64.Ri [doi:10.1250/ast.41.196]

\section{INTRODUCTION}

Cortical structures in the adult human brain were previously considered to be non-elastic; however, recent studies have revealed that the adult brain can be reorganized based on its surrounding environment and behavior. Reorganization in the human auditory cortex generally occurs in a direction that is preferable for fulfilling demands [1,2]; however, it may also induce pathological phenomena in the auditory system such as tinnitus [3]. Therefore, it appears to be important to develop suitable neurorehabilitation approaches to treat maladaptive reorganization in the auditory system [4]. I herein report maladaptive reorganization in the human auditory cortex of individuals who regularly listened to loud music via portable music players [5] and in the unilateral tinnitus patients [6]. I also introduce our neurorehabilitation approaches for sudden sensorineural hearing loss (SSHL) [7] based on cortical plasticity in the auditory system.

\section{EFFECTS OF LOUD MUSIC VIA PORTABLE MUSIC PLAYERS ON THE AUDITORY EVOKED RESPONSES}

Portable music players and smart phones are daily used

*e-mail: okamoto@iuhw.ac.jp by numerous adolescents in order to listen to music in noisy public environments; however, the effects of portable music player usage for a long time and at a high volume remain elusive $[8,9]$. We used a non-invasive electrophysiological technique called magnetoencephalography (MEG) and measured the auditory evoked fields in two experimental groups. The "User" group consisted of 13 young subjects who had listened to music via portable music players regularly for at least the last two years. Their comfortable volume setting for listening to music was $80.9 \pm 10.2 \mathrm{~dB}$ in A-weighted sound pressure level in a sound-proof room. The "Non-User" group consisted of 13 young subjects who had not yet used portable music players. The findings of pure tone audiometry, a speech test, and hearing in a noise test [10] were similar between the two groups.

We measured population-level frequency tuning of the two groups as described in the previous studies [11-13]. Auditory evoked magnetic fields were elicited by tonal test stimuli (TS), which were presented either in isolation or embedded in band-eliminated noise (BEN) (c.f. Fig. 1). Simultaneously presented BEN was broadband noise containing eliminated bands with widths of either a quarter, half, or one critical band (CB) [14] centered at the embedded TS frequency. Neurons of the auditory cortex activated by TS or BEN overlap partially, and the degree of 

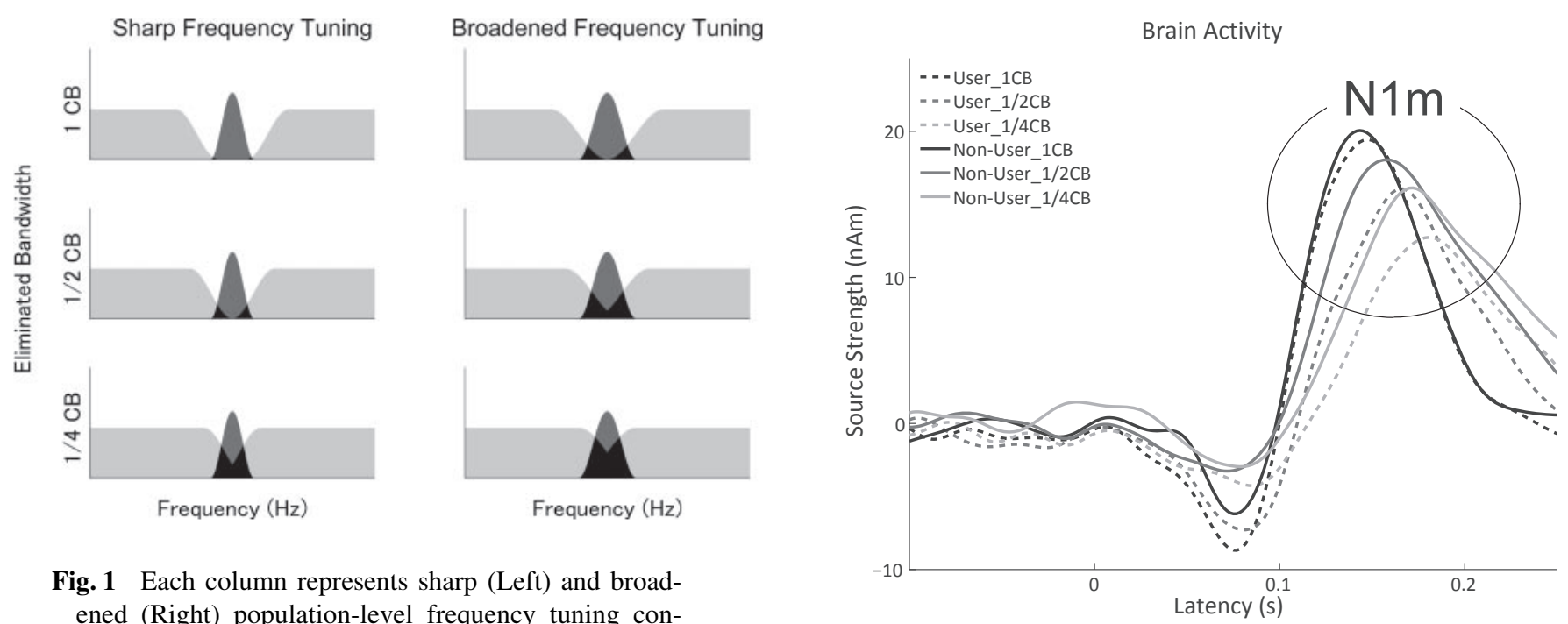

Fig. 1 Each column represents sharp (Left) and broadened (Right) population-level frequency tuning conditions. Each row represents wide 1 critical band (CB) (Top), 1/2 CB (middle), and 1/4 CB (bottom) bandeliminated noise (BEN) condition. Light gray, dark gray, and black colored areas represent neural activity elicited by BEN, test stimulus (TS), and both BEN and TS, respectively. The N1m response elicited by TSonset is represented by dark gray areas because neural activity represented by the black areas has been already masked by BEN (modified from Okamoto et al., 2011 [5]).

overlap depends on both the width of the eliminated band of BEN and the sharpness of population-level frequency tuning. Narrower BEN and broader frequency tuning would cause larger overlaps and result in smaller auditory evoked responses elicited by TS than BEN with wider eliminated bands and sharper frequency tuning. We measured the auditory evoked fields under distracted listening conditions. During the MEG measurement, participants had to perform a visual task in order to avoid paying attention to the auditory modality.

Auditory evoked fields were measured with a wholehead MEG system in a silent magnetically shielded room. The auditory evoked fields elicited by TS were selectively averaged for each BEN condition (BEN_1/4CB, BEN $1 / 2 \mathrm{CB}$, BEN_1CB, and no BEN). In the analysis of N1m response, which is a major deflection of the auditory evoked response originating from auditory belt area [15], the noBEN condition was used for estimations of $\mathrm{N} 1 \mathrm{~m}$ dipole source locations and orientations. Estimated sources were fixed in their locations and orientations as a spatial filter, and the corresponding source strength waveforms were calculated for each BEN condition (BEN_1/4CB, BEN $1 / 2 \mathrm{CB}$, and BEN_1CB) (c.f. Fig. 2). N1m source strengths were normalized with respect to the mean $\mathrm{N} 1 \mathrm{~m}$ source strength averaged across the BEN_1/4CB, BEN_1/2CB, and BEN_1CB conditions for each hemisphere and participant. Thereafter, normalized $\mathrm{N} 1 \mathrm{~m}$ source strengths were analyzed via repeated-measures of analysis of variances

(ANOVAs) using two factors: BEN-TYPE (BEN_1/4CB, BEN_1/2CB, and BEN_1CB) and GROUP ("User" vs. "Non-User"). The repeated-measures ANOVA revealed a significant main effect for BEN-TYPE $(F(2,50)=46.1$, $p<0.001$ ), and a significant interaction between GROUP and BEN-TYPE $(F(2,100)=3.7, p<0.03)$. Narrower BEN caused a smaller N1m source strength ratio in both groups; however, this effect was more obvious in the "User" group than in the "Non-User" group. This finding indicated that population-level frequency tuning was broadened significantly more in the "User" group than in the "Non-User" group [5]. The broadened frequency tuning may be a prodromal stage to forthcoming pathological symptoms such as tinnitus and hearing loss.

\section{POPULATION-LEVEL FREQUENCY TUNING IN TINNITUS PATIENTS}

Subjective tinnitus is a phantom auditory sensation without any external sound source [16]. Human neuroimaging studies have revealed that subcortical and cortical activities play an important role in the perception of tinnitus [17,18]. However, most of previous studies have investigated the auditory neural activity of humans or animals with hearing loss; therefore, it has yet to be clarified whether the findings obtained in these studies reflect hearing loss, subjective tinnitus, or a combination of the two.

We hypothesized that subjective tinnitus perception is related to broadened frequency tuning within the human 
auditory cortex irrespective of hearing loss. Therefore, we objectively measured population-level frequency tuning in seven unilateral tinnitus patients with similar hearing levels between their ears as described in the previous section. We used a pure tone with each participant's tinnitus frequency as TS either in isolation or embedded in BEN. TS and BEN were delivered monaurally and diotically, respectively. We prepared four conditions: TS with BEN presented to the tinnitus ear ("Noisy_Tinnitus") or non-tinnitus ear ("Noisy_Non-Tinnitus"), and TS presented in silence to the tinnitus ear ("Silent_Tinnitus") or non-tinnitus ear ("Silent_Non-Tinnitus").

The experimental protocol was similar to the previous section. Epochs of data elicited by TS were averaged selectively for each condition after the rejection of artifact epochs. N1m source strengths elicited by TS in each condition were analyzed via repeated-measures ANOVA using BEN ("Noisy" vs. "Silent") and EAR ("Tinnitus" vs. "Non-Tinnitus") as factors. The N1m source strengths elicited in the "Noisy" condition were normalized with respect to the N1m source strengths elicited in the "Silent" condition for each hemisphere and participant. The normalized N1m source strengths in each EAR condition are presented with 95\% confidence intervals in Fig. 3. A paired $t$-test applied to the normalized $\mathrm{N} 1 \mathrm{~m}$ source strengths revealed a significant difference between "Tinnitus" and "Non-Tinnitus" $(t(1,6)=3.16 ; p<0.02)$.

The findings obtained showed that $\mathrm{N} 1 \mathrm{~m}$ decrements caused by BEN were more prominent when sounds were delivered to the tinnitus ear than to the non-tinnitus ear, suggesting that population-level frequency tuning was broader when sounds were delivered to the tinnitus ear than to the non-tinnitus ear (c.f. Fig. 1). The participants had similar hearing levels between their ears. Therefore, broadened population-level frequency tuning appeared to

\section{Normalized N1m responses}

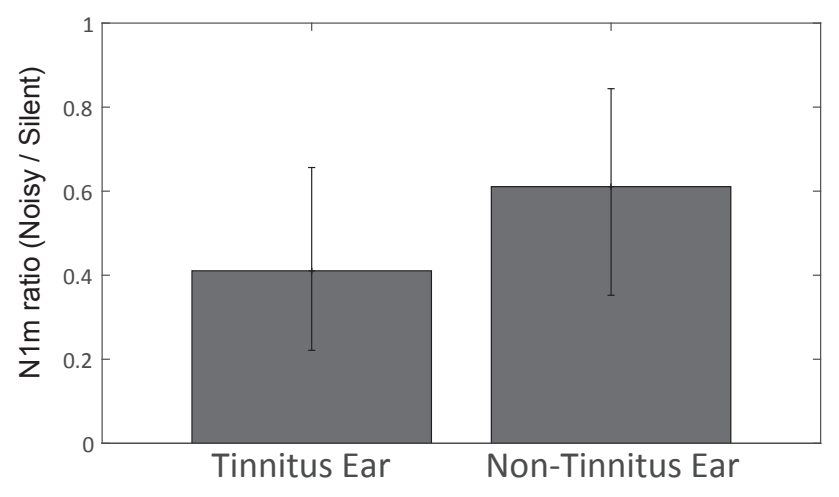

Fig. 3 Group means of $\mathrm{N} 1 \mathrm{~m}$ source strength ratios (Noisy/Silent) elicited in tinnitus (Left) and nontinnitus (Right) ears. Error bars denote $95 \%$ confidence intervals. (modified from Sekiya et al., 2017 [6]). be related to the existence of subjective tinnitus, and not to hearing loss. Broader population-level frequency tuning in the tinnitus ear supported the hypothesis that pathological alterations in inhibitory neural networks in the central auditory pathway may play an important role in the emergence and maintenance of subjective tinnitus symptoms [19-21].

\section{CONSTRAINT INDUCED SOUND THERAPY AGAINST SUDDEN SENSORINEURAL HEARING LOSS (SSHL)}

SSHL is an idiopathic condition characterized by the rapid loss of hearing without a clear pathological cause [22]. We focused on cortical reorganization initiated by hearing loss [23,24] and developed a new treatment strategy named constraint induced sound therapy (CIST) for SSHL [7,25]. We tried to treat SSHL by preventing maladaptive reorganization in the human auditory cortex. In this study, we plugged the outer ear canal of the healthy ear of SSHL patients and forced them to use the hearing loss ear (Fig. 4). Moreover, we asked them to listen to classical music via their hearing loss ear for 6 hours per day during their hospitalization. Treatment outcomes were evaluated by comparing the hearing threshold levels of the two SSHL groups. One group was the "control" group $(N=31)$ who merely received the normal corticosteroid therapy, while the "target" group $(N=22)$ received CIST in addition to the corticosteroid therapy. Treatment outcomes were evaluated by comparing the hearing threshold levels and brain activities of the two SSHL groups.

Participants performed pure tone audiometric tests at the entrance examination (1st measurement), the discharge from hospital (2nd measurement), and visiting us as outpatients (3rd measurement). Auditory evoked neural responses in 6 SSHL patients in the "target" group were measured by MEG. They participated in the MEG sessions before and after CIST. During MEG measurements, we used a monaurally presented $40-\mathrm{Hz}$ amplitude-modulated

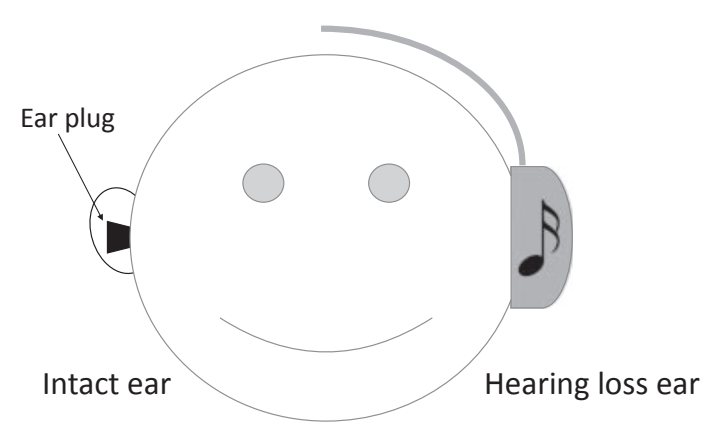

Fig. 4 Schematic illustration of constraint-induced sound therapy (CIST). The intact ear was plugged in order to promote the usage of the hearing loss ear. Music was presented to the hearing loss ear. 
TS to the hearing loss or healthy ear. During MEG measurements, the TS was delivered at an intensity of $45 \mathrm{~dB}$ above individual sensation thresholds in the healthy ear and a loudness matched TS was delivered to the hearing loss ear. The $\mathrm{N} 1 \mathrm{~m}$ source strengths were analyzed in a similar manner to previous studies. In order to investigate the degree of cortical reorganization, we calculated the laterality indices (LIs) of neural activities in the ipsilateral and contralateral hemispheres to the stimulated ear. LI was calculated as follows: $(A-B) /(A+B), A=$ source strength elicited in the contralateral hemisphere, $B=$ source strength elicited in the ipsilateral hemisphere. It is known that normal-hearing people generally exhibit positive values of LIs, indicating the contralateral hemispheric dominance of neural activity in the auditory cortex.

No adverse events associated with CIST occurred. The mean hearing threshold differences between the hearing loss and healthy ears averaged across all frequencies (125, $250,500,1,000,2,000,4,000$, and $8,000 \mathrm{~Hz}$ ) were similar between groups in the 1st audiometric examination (Fig. 5 left). However, they significant differed in the 2nd (Fig. 5 middle) and 3rd examinations (Fig. 5 right). The degree of hearing recovery in the control group was similar to that reported in an epidemiological study [26]. Participants in the "target" group recovered significantly better than those in the "control" group. Therefore, the significantly better recovery of hearing thresholds in the "target" group than in the "control" group reflects the effects of CIST against SSHL. Figure 6 shows the LIs of N1m responses in six "target" group patients. In the 1st MEG recording (before

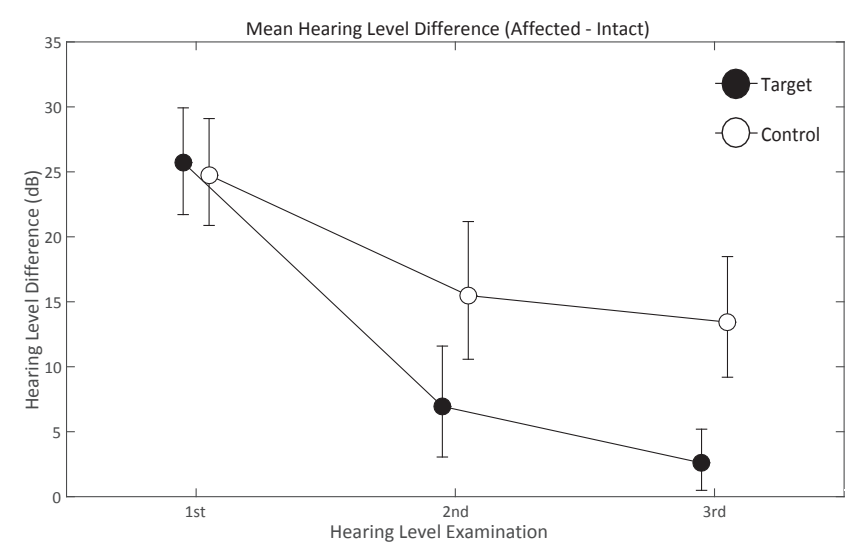

Fig. 5 Mean hearing threshold differences between hearing loss and intact ears in the 1st (left: entering hospital), 2nd (center: leaving hospital), and 3rd (right: first visit as outpatient after discharge) pure tone audiometric examinations. The filled circles indicate the "Target" group that received constraint-induced sound therapy (CIST) and standard corticosteroid therapy. The open circles indicate the "Control" group that merely received standard corticosteroid therapy. Error bars denote $95 \%$ confidence intervals (modified from Okamoto et al., 2014 [7]).

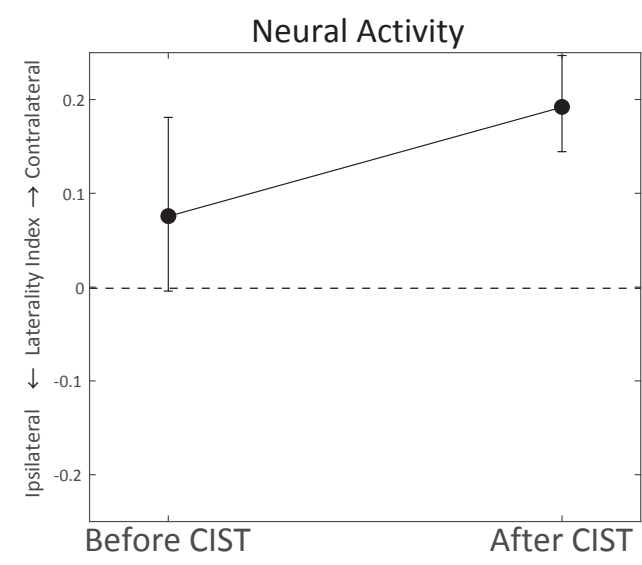

Fig. 6 Laterality indices (LIs) of the N1m responses before and after constraint-induced sound therapy (CIST). Error bars denote 95\% confidence intervals (modified from Okamoto et al., 2014 [7]).

the treatment), the LIs of $\mathrm{N} 1 \mathrm{~m}$ were not significantly different from 0 , suggesting that the neural activity elicited by monaural stimulation was similar between ipsilateral and contralateral hemispheres as previously reported $[23,27]$. However, after CIST, the LIs of N1m responses showed contralateral hemispheric dominancy as observed in normal hearing people.

In an animal study [28], after loud noise exposure, cats housed in an enriched acoustic environment showed betterpreserved tonotopic maps in the primary auditory cortex than those housed in a quiet environment. In our study, CIST extensively activated auditory neurons corresponding to the affected ear, while neural activity corresponding to the intact ear was reduced due to the ear plug. We observed a significantly greater improvement in hearing thresholds in the "target" group than that in the control group. Moreover, the neuroimaging data indicated the reversal of SSHL-related maladaptive reorganization in the primary and non-primary auditory cortex. The addition of our safe and cost-effective CIST to standard corticosteroid treatment appears to be beneficial for SSHL patients.

\section{CONCLUSION}

Neurophysiological studies revealed that the auditory cortex can be maladaptively reorganized based on hearing impairments. We visualized that the neural activity could be modulated by listening to loud music via portable music players [5] and by the emergence of tinnitus [6]. Visualization of the healthy and pathological brain activity by non-invasive neuroimaging techniques appears to contribute to the development of a new therapeutic approach for those affected. In fact, we developed rehabilitation approaches that reduce or prevent maladaptive cortical plasticity and improve the quality of life of tinnitus [29,30] and SSHL patients [7,25]. The rehabilitation 
approaches based on the neurophysiological findings will become more important in future.

\section{REFERENCES}

[1] C. Pantev, R. Oostenveld, A. Engelien, B. Ross, L. E. Roberts and M. Hoke, "Increased auditory cortical representation in musicians," Nature, 392(6678), 811-814 (1998).

[2] C. Gaser and G. Schlaug, "Brain structures differ between musicians and non-musicians," J. Neurosci., 23, 9240-9245 (2003).

[3] W. Muhlnickel, T. Elbert, E. Taub and H. Flor, "Reorganization of auditory cortex in tinnitus," Proc. Natl. Acad. Sci. USA, 95, 10340-10343 (1998).

[4] T. Elbert and S. Heim, "A light and a dark side," Nature, 411(6834), 139 (2001).

[5] H. Okamoto, H. Teismann, R. Kakigi and C. Pantev, "Broadened population-level frequency tuning in human auditory cortex of portable music player users," PLOS ONE, 6(3), e17022 (2011).

[6] K. Sekiya, M. Takahashi, S. Murakami, R. Kakigi and H. Okamoto, "Broadened population-level frequency tuning in the auditory cortex of tinnitus patients," J. Neurophysiol., 117, 1379-1384 (2017).

[7] H. Okamoto, M. Fukushima, H. Teismann, L. Lagemann, T. Kitahara, H. Inohara, R. Kakigi and C. Pantev, "Constraintinduced sound therapy for sudden sensorineural hearing loss: Behavioral and neurophysiological outcomes," Sci. Rep.-Uk, 4, 3927 (2014).

[8] F. Zhao, V. K. Manchaiah, D. French and S. M. Price, "Music exposure and hearing disorders: An overview," Int. J. Audiol., 49, 54-64 (2010).

[9] A. Axelsson, U. Rosenhall and G. Zachau, "Hearing in 18year-old swedish males," Scand. Audiol., 23, 129-134 (1994).

[10] M. Nilsson, S. D. Soli and J. A. Sullivan, "Development of the hearing in noise test for the measurement of speech reception thresholds in quiet and in noise," J. Acoust. Soc. Am., 95, 1085-1099 (1994).

[11] H. Okamoto, H. Stracke, C. H. Wolters, F. Schmael and C. Pantev, "Attention improves population-level frequency tuning in human auditory cortex," J. Neurosci., 27, 10383-10390 (2007).

[12] H. Okamoto, H. Stracke, P. Zwitserlood, L. E. Roberts and C. Pantev, "Frequency-specific modulation of population-level frequency tuning in human auditory cortex," BMC Neurosci., 10, 1 (2009).

[13] M. Sams and R. Salmelin, "Evidence of sharp frequency tuning in the human auditory cortex," Hear. Res., 75, 67-74 (1994).

[14] E. Zwicker and H. Fastl, Psychoacoustics: Facts and Models, 2nd ed. (Springer, Berlin/New York, 1999).

[15] R. Näätänen and T. Picton, "The N1 wave of the human electric and magnetic response to sound: A review and an analysis of the component structure," Psychophysiology, 24, 375-425 (1987).

[16] J. J. Eggermont and L. E. Roberts, "The neuroscience of tinnitus," Trends Neurosci., 27, 676-682 (2004).

[17] T. Ueyama, T. Donishi, S. Ukai, Y. Yamamoto, T. Ishida, S. Tamagawa, M. Hotomi, K. Shinosaki, N. Yamanaka and Y. Kaneoke, "Alterations of regional cerebral blood flow in tinnitus patients as assessed using single-photon emission computed tomography," PLOS ONE, 10(9), e0137291 (2015).

[18] N. Weisz, C. Wienbruch, K. Dohrmann and T. Elbert, "Neuromagnetic indicators of auditory cortical reorganization of tinnitus," Brain, 128, 2722-2731 (2005).

[19] E. Diesch, M. Andermann, H. Flor and A. Rupp, "Interaction among the components of multiple auditory steady-state responses: Enhancement in tinnitus patients, inhibition in controls," Neuroscience, 167, 540-553 (2010).

[20] E. Diesch, M. Struve, A. Rupp, S. Ritter, M. Hulse and H. Flor, "Enhancement of steady-state auditory evoked magnetic fields in tinnitus," Eur. J. Neurosci., 19, 1093-1104 (2004).

[21] A. Kral and V. Majernik, "On lateral inhibition in the auditory system," Gen. Physiol. Biophys., 15, 109-127 (1996).

[22] National Institute of Health, Sudden Deafness, Vol. 00-4757 (NIH Publication, Bethesda, 2000).

[23] L. P. H. Li, A. S. Shiao, K. C. Chen, P. L. Lee, D. M. Niddam, S. Y. Chang and J. C. Hsieh, "Neuromagnetic index of hemispheric asymmetry prognosticating the outcome of sudden hearing loss," PLoS ONE, 7(4), e35055 (2012).

[24] M. Suzuki, H. Kouzaki, Y. Nishida, A. Shiino, R. Ito and H. Kitano, "Cortical representation of hearing restoration in patients with sudden deafness," Neuroreport, 13, 1829-1832 (2002).

[25] K. Sekiya, M. Fukushima, H. Teismann, L. Lagemann, R. Kakigi, C. Pantev and H. Okamoto, "Neuro-rehabilitation approach for sudden sensorineural hearing loss," J. Vis. Exp., (107), e53264 (2016).

[26] F. M. Byl, Jr., "Sudden hearing loss: Eight years' experience and suggested prognostic table," Laryngoscope, 94(5 Pt 1), 647-661 (1984).

[27] D. Bilecen, E. Seifritz, E. W. Radu, N. Schmid, S. Wetzel, R. Probst and K. Scheffler, "Cortical reorganization after acute unilateral hearing loss traced by fMRI," Neurology, 54, 765767 (2000)

[28] A. J. Norena and J. J. Eggermont, "Enriched acoustic environment after noise trauma reduces hearing loss and prevents cortical map reorganization," J. Neurosci., 25, 699-705 (2005).

[29] H. Okamoto, H. Stracke, W. Stoll and C. Pantev, "Listening to tailor-made notched music reduces tinnitus loudness and tinnitus-related auditory cortex activity," Proc. Natl. Acad. Sci. USA, 107, 1207-1210 (2010).

[30] H. Stracke, H. Okamoto and C. Pantev, "Customized notched music training reduces tinnitus loudness," Commun. Integr. Biol., 3, 274-277 (2010) 